\title{
Estigma social e as famílias de doentes com tuberculose: um estudo a partir das análises de agrupamento e de correspondência múltipla
}

\author{
Social stigma and the families of patients with tuberculosis: \\ a study based on cluster and multiple correspondence analysis
}

\author{
Michelle Mosna Touso ${ }^{1}$ \\ Marcela Paschoal Popolin ${ }^{1}$ \\ Juliane de Almeida Crispim ${ }^{1}$ \\ Isabela Moreira de Freitas ${ }^{1}$ \\ Ludmila Barbosa Bandeira Rodrigues ${ }^{1}$ \\ Mellina Yamamura ${ }^{1}$ \\ Ione Carvalho Pinto ${ }^{1}$ \\ Aline Aparecida Monroe ${ }^{1}$ \\ Pedro Fredemir Palha ${ }^{1}$ \\ Antônio Sérgio Ferraudo ${ }^{2}$ \\ Tereza Cristina Scatena Villa ${ }^{1}$ \\ Ricardo Alexandre Arcêncio ${ }^{1}$
}

\footnotetext{
${ }^{1}$ Departamento de Enfermagem MaternoInfantil e Saúde Pública, Escola de Enfermagem de Ribeirão Preto, Universidade de São Paulo. Av. Bandeirantes 3900, Vila Monte Alegre. 14040-902 Ribeirão Preto SP Brasil. ricardo@eerp.usp.br ${ }^{2}$ Departamento de Ciências Exatas, Universidade Estadual Paulista Júlio de Mesquita Filho.
}

\begin{abstract}
The social stigma associated with TB is a challenge facing management of the area of public health care. The aim of this study was to investigate the social stigma in families of patients with $T B$ and identify the profile of those who are affected by the event in relation to socioeconomic and demographic conditions. It is a cross-sectional study that was conducted in 2011 in the city of Ribeirão Preto, state of São Paulo, Brazil, with a sample of 110 individuals. The data were analyzed using the univariate descriptive technique and cluster and multiple correspondence assessment. The stigmatized groups tend to have lower scholarity, incipient access to the media and little understanding about TB, as opposed to those that have higher educational levels, continuous access to the media, consider themselves well informed and show proactive attitudes to deal with the disease. The identification of varied profiles highlights the need to develop health interventions to cater to the singularities of families with respect to the social stigma of the disease.
\end{abstract}

Key words Social stigma, Tuberculosis, Family
Resumo O estigma social da tuberculose é um desafio que se coloca à gestão do cuidado na área da saúde pública. O objetivo deste estudo foi investigar o estigma social em famílias de doentes com TB e identificar o perfil daquelas que são acometidas pelo evento em relação às condições socioeconômicas e demográficas. Trata-se de um estudo transversal realizado no município de Ribeirão Preto, São Paulo, Brasil, em 2011, com uma amostra de 110 sujeitos. Os dados foram analisados mediante técnica descritiva univariada $e$ análises de agrupamento e de correspondência múltipla. Os grupos com estigma apresentaram baixa escolaridade, acesso incipiente aos meios de informação e pouca mobilização referente à compreensão sobre a doença, diferenciando-se dos demais grupos que apresentam comportamentos e atitudes positivas, com niveis de escolaridade mais elevados, acesso contínuo aos meios de informação e se dizem conhecedores da TB. A identificação de diferentes perfis aponta para necessidade de serem desenvolvidas ações em saúde sensiveis às singularidades das famílias no que se refere ao estigma social da doença.

Palavras-chave Estigma social, Tuberculose, Família 


\section{Introdução}

No mundo, a tuberculose (TB) ainda é um problema de saúde pública que vem preocupando as autoridades da área da saúde. Aproximadamente um terço da população mundial está infectado pelo Mycobacterium tuberculosis e em risco de desenvolver a doença. Dentre os 22 países responsáveis por $80 \%$ de todos os casos de TB, o Brasil ocupa a $17^{\text {a }}$ posição em relação ao número e a $111^{\text {a }}$ quanto ao coeficiente de incidência $(\mathrm{CI})^{1}$.

No país, em 2012, foram notificados 70.047 casos novos da doença, o que equivale ao CI de 36,1/100.000 habitantes. Segundo a Organização Mundial da Saúde (OMS), o Brasil já alcançou a meta de reduzir pela metade a taxa de mortalidade por TB, quando comparada ao ano de $1990^{2}$.

A TB é um dos agravos de saúde influenciado por determinantes sociais e demonstra relação direta com a pobreza e a exclusão, no qual o estigma tem sido considerado um indicador importante desta relação ${ }^{3,4}$. O termo estigma foi originalmente descrito por Goffman ${ }^{5}$ como um atributo indesejável ou depreciativo aos olhos da sociedade, podendo ser uma marca física ou social de conotação negativa, que leva o indivíduo à marginalização e/ou à exclusão de suas relações sociais ${ }^{6}$.

Para os autores ${ }^{6}$, o estigma social pode causar forte impacto na vida da pessoa o sofre e em sua família. Sob esse aspecto, pesquisas ${ }^{7-11}$ apontam que o principal efeito do estigma associado a TB, nos países em desenvolvimento, é o isolamento social do doente na comunidade e na família, no qual o sujeito é forçado a comer e a dormir separadamente dos demais membros ${ }^{12}$.

Cabe destacar que registros históricos mostram que o estigma da TB na dinâmica familiar não é uma questão recente. No final do século XIX, a morte por TB na família era estigmatizante, pois a doença estava relacionada a algum defeito hereditário e/ou à pobreza. Nesta época, $\mathrm{o}$ doente era desqualificado para o casamento, para o exercício de determinadas atividades e visto como uma ameaça na vida de outros familiares ${ }^{13}$.

Hoje, a representação da doença na família conserva muitas das imagens que fizeram da TB uma das doenças mais temidas em todos os tempos. Segundo autores ${ }^{14}$, diante do adoecimento a família reage de forma peculiar e própria, uma vez que este processo é determinado por múltiplas variáveis. Portanto, são relevantes estudos que apresentem os determinantes associados ao estigma da TB, a fim de se estabelecer o perfil das famílias acometidas pelo evento.
Desta forma, atribui-se a importância da família para a investigação, haja vista que o estigma social na dinâmica familiar pode levar a atrasos na busca por cuidados e afetar a adesão ao tratamento, sendo barreiras consideráveis para o controle da $\mathrm{TB}^{8,11,15}$. Assim, propôs-se investigar o estigma social em famílias de doentes com TB e identificar o perfil daquelas que são acometidas por este evento em relação às condições socioeconômicas e demográficas.

\section{Métodos}

Trata-se de um estudo transversal realizado em Ribeirão Preto, município prioritário para o controle da TB no país ${ }^{16}$. Localizado na região Nordeste do Estado de São Paulo, a 313km da capital estadual e a 706km de Brasília, Ribeirão Preto possui um território de $650 \mathrm{~km}^{2}$, com população urbana, estimada para 2011, de 604.682 habitantes. A cidade apresenta Índice de Desenvolvimento Humano (IDH) de 0,85 , apesar de $89,2 \%$ dos moradores estarem acima da linha da pobreza. No tocante ao Índice Paulista de Responsabilidade Social (IPRS), o município se enquadra no grupo 1 , apresentando bons indicadores sociais e econômicos ${ }^{17,18}$.

$\mathrm{O}$ atendimento aos doentes de TB é centralizado nos ambulatórios de referência com Programas de Controle da Tuberculose (PCT), distribuídos em cinco distritos sanitários (leste, oeste, norte, sul e central). Estes serviços operam com equipes especializadas, compostas minimamente por um médico, dois auxiliares de enfermagem e uma enfermeira, que realizam atividades voltadas para diagnóstico, manejo clínico dos casos e de seus comunicantes, consulta médica de controle e Tratamento Diretamente Observado (TDO), que, em 2010, cobriu 93\% dos doentes de TB. Em relação ao desfecho do tratamento, o município atingiu $85,1 \%$ de cura, $3,9 \%$ de abandono, $1 \%$ de óbito por TB, sendo que $2,6 \%$ dos casos não tinham informações sobre o encerramento ${ }^{19}$.

A população base do estudo foi composta por familiares comunicantes dos doentes de TB, residentes no município, com idade igual ou superior a 18 anos e que consentiram participar voluntariamente da pesquisa. Excluíram-se os familiares que estivessem em instituições de longa permanência ou quando não fossem localizados no domicílio após três tentativas dos entrevistadores.

Para o cálculo da amostra, foi feito um levantamento do número de doentes de TB diagnosti- 
cados entre 01 de janeiro de 2010 a 31 de junho de 2011, nas bases de dados do Sistema Estadual de Notificação da Tuberculose (TBWEB), identificando-se um total de 155 sujeitos. Assumindo nível de confiança de 95\% e erro amostral tolerável de 5\% estimou-se uma amostra mínima de 110 sujeitos. Assim, para cada caso-índice de TB registrado no sistema, foi incluído, por conveniência, um familiar.

Os dados foram coletados no período de julho a agosto de 2011 por meio de entrevistas com os familiares dos doentes de TB, utilizando um instrumento elaborado com base em escalas validadas para avaliar o estigma da TB na Nicarágua, Sul da Tailândia, Malawi, Bangladesh e Índia ${ }^{20-22}$. Os dez itens do instrumento no Brasil abrangem os seguintes domínios-chave: medo de transmitir a doença, atitudes associadas à vergonha, julgamento e revelação da doença. Esses itens foram avaliados por dez juízes, especialistas na temática, que verificaram a adequação conceitual dos atributos, adotando-se como critério de pertinência do item, $80 \%$ de concordância. Realizou-se o teste piloto com dez sujeitos, que confirmou a sensibilidade cultural do instrumento na população de referência.

O plano analítico se deu em dois momentos, inicialmente procedeu-se a análise descritiva das condições socioeconômicas e demográficas, elegendo-se as seguintes variáveis: sexo, idade, escolaridade, estado civil, religião, situação profissional, renda e número de residentes no domicílio. Em relação ao estigma, este foi medido utilizando as dez questões do instrumento. Para tal, os itens da escala tipo Likert (cinco pontos) foram dicotomizados em sim e não. Na sequência, realizou-se a somatória das respostas para cada item, dividindo-a pelo total da amostra e multiplicando por 100 (Tabela 1).

Posteriormente, para identificação de perfis de famílias, recorreu-se à estatística multivariada, sendo esta etapa analítica composta pela Análise de Agrupamento (AA) por níveis hierárquicos e Análise de Correspondência Múltipla (ACM).

Cabe ressaltar que a $\mathrm{AA}$ por níveis hierárquicos possibilitou identificar a estrutura de grupos, a qual foi considerada como medida de semelhança entre os sujeitos, sendo a distância euclidiana e o algoritmo de Ward considerados como método de ligação. Essa técnica deu origem ao dendrograma (Figura 1) e permitiu o agrupamento dos sujeitos "semelhantes" de acordo com as variáveis investigadas. Segundo Mingoti ${ }^{23}$ através do dendrograma é possível verificar a similaridade entre as observações, considerando que a
Tabela 1. Distribuição percentual das condições socioeconômicas e demográficas dos familiares, Ribeirão Preto (SP), 2011.

\begin{tabular}{|c|c|c|}
\hline Variáveis & $\begin{array}{c}\mathbf{n} \\
(110)\end{array}$ & $\%$ \\
\hline \multicolumn{3}{|l|}{ Sexo } \\
\hline Feminino & 85 & 77,3 \\
\hline Masculino & 25 & 22,7 \\
\hline \multicolumn{3}{|l|}{ Idade (anos) } \\
\hline 18 a 38 & 33 & 30,0 \\
\hline 39 a 59 & 50 & 45,4 \\
\hline$\geq 60$ & 27 & 24,6 \\
\hline \multicolumn{3}{|l|}{ Escolaridade } \\
\hline Até o Ensino Fundamental Incompleto & 58 & 52,7 \\
\hline Fundamental Completo & 16 & 14,6 \\
\hline Médio Completo & 32 & 29,0 \\
\hline Superior Completo & 4 & 3,7 \\
\hline \multicolumn{3}{|l|}{ Estado Civil } \\
\hline Solteiro(a) & 26 & 23,7 \\
\hline Casado(a) & 66 & 60,0 \\
\hline Divorciado(a) & 6 & 5,4 \\
\hline Viúvo & 12 & 10,9 \\
\hline \multicolumn{3}{|l|}{ Religião } \\
\hline Não tem & 18 & 16,3 \\
\hline Católica & 57 & 51,9 \\
\hline Evangélica & 23 & 20,9 \\
\hline Espírita & 7 & 6,3 \\
\hline Outra & 5 & 4,6 \\
\hline \multicolumn{3}{|l|}{ Situação Profissional } \\
\hline Trabalha & 72 & 65,5 \\
\hline Desempregado & 15 & 13,6 \\
\hline Não Trabalha & 23 & 20,9 \\
\hline \multicolumn{3}{|l|}{ Renda $\left(\mathrm{SM}^{*}\right)$} \\
\hline$<1$ & 26 & 23,7 \\
\hline 1 a 2,5 & 49 & 44,6 \\
\hline 2,6 a 5 & 28 & 25,4 \\
\hline 5,1 a 10 & 6 & 5,4 \\
\hline$>10$ & 1 & 0,9 \\
\hline \multicolumn{3}{|l|}{ Residentes } \\
\hline$\leq 3$ habitantes & 46 & 41,8 \\
\hline$>3$ habitantes & 64 & 58,2 \\
\hline
\end{tabular}

Fonte: Cálculo dos autores. * SM - salário mínimo vigente na ocasião da coleta de dados era de R\$ 600,00.

menor distância entre elas corresponde ao maior nível de semelhança.

Após a identificação dos grupos, utilizou-se o teste multivariado $\mathrm{T}^{2}$ de Hotelling, para verificar a diferença estatística entre os mesmos ${ }^{24}$. Fixouse em $5 \%$ a probabilidade de erro tipo I. A identificação dos grupos gerou uma nova variável entre estes e os sujeitos. Essa variável foi utilizada como complementar na Análise de Correspondência Múltipla (ACM), aplicada para buscar a 


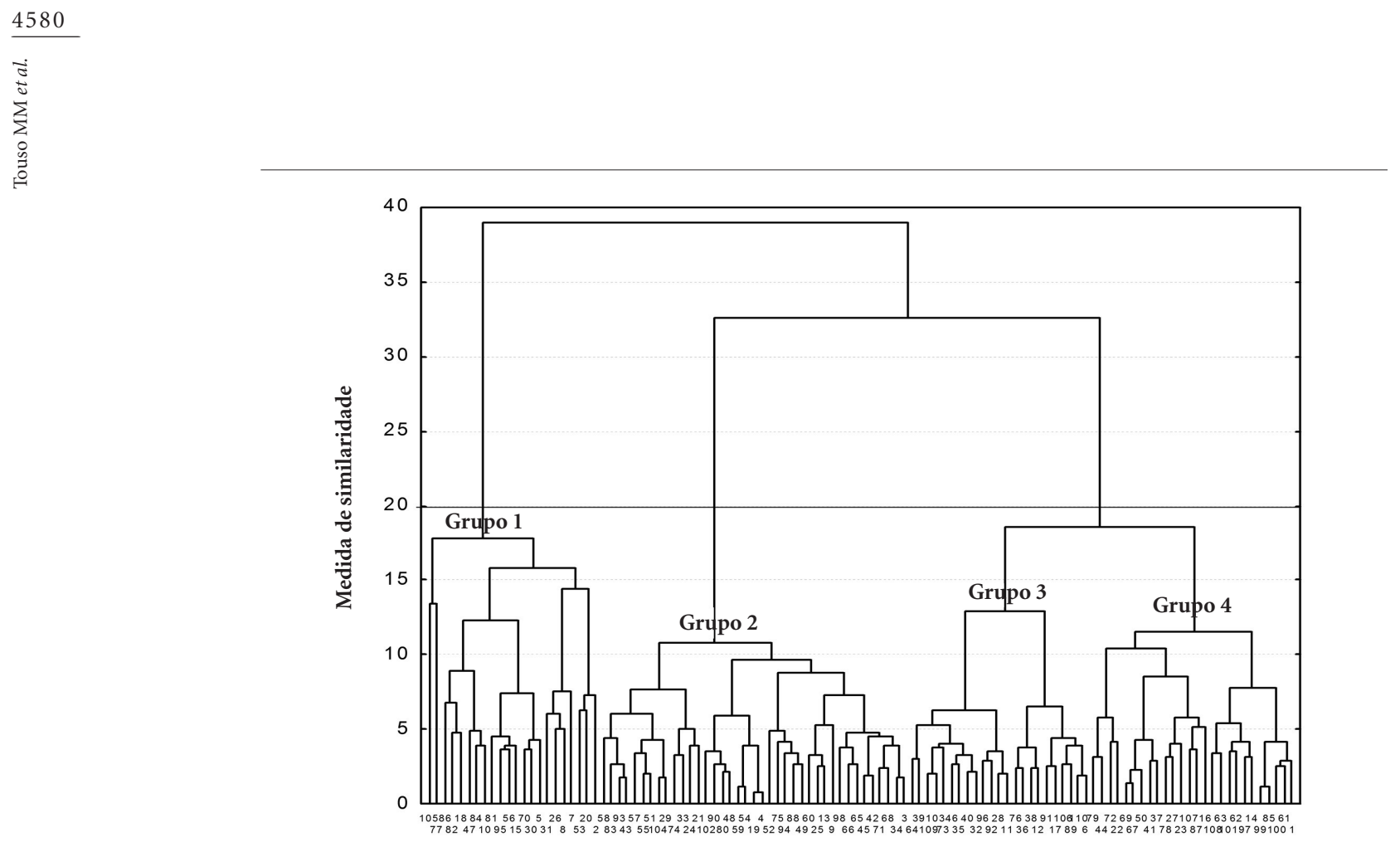

Figura 1. Dendrograma resultante da análise de agrupamento obtido com as respostas das famílias quanto ao estigma, Ribeirão Preto (SP), 2011.

estrutura de equivalência no conjunto inicial de variáveis.

Para a aplicação da ACM seguiram-se os passos descritos por autores ${ }^{25}$, na qual a tabulação das respostas do questionário gerou uma matriz em que as linhas correspondem aos sujeitos e, as colunas às variáveis. Posteriormente, essa matriz foi transformada em Tabela Disjuntiva Completa (TDC). Nesta tabela, as colunas representam as características das variáveis, em que a intersecção da linha "i" com a coluna "i" encontra-se $x_{i j}$ que vale 0 ou 1 , indicando que o sujeito " $i$ " possui ou não a característica “ $j$ ”.

O mapa perceptual foi formado utilizando essa técnica, estando a representação visual das variáveis em duas ou mais dimensões. Cada variável tem uma posição espacial no mapa perceptual, variáveis percebidas como similares ou associadas são alocadas em pontos próximos no mapa, ao passo que aquelas percebidas como não similares são representadas em pontos distantes. Ou seja, a proximidade indica a correspondência entre as categorias representadas nas linhas e nas colunas da tabela. Ressalta-se que o elemento linha ou coluna influi na construção dos eixos por meio de sua inércia, em relação ao centro de gravidade, sendo esta inércia a variância do conjunto de dados ${ }^{26}$.

A partir da ACM, foi possível extrair as dimensões mais representativas em relação à inér- cia, que no estudo correspondeu às duas primeiras, sendo considerando como critério para seleção das variáveis a contribuição das mesmas para a inércia (variância). No curso das análises, foram consideradas 18 variáveis, com um total de 89 modalidades de resposta, sendo as socioeconômicas, demográficas e de estigma definidas como Ativa e a do grupo como Suplementar ${ }^{26}$.

Salienta-se que o projeto de pesquisa foi aprovado pelo Comitê de Ética em Pesquisa da Escola de Enfermagem de Ribeirão Preto da Universidade de São Paulo.

\section{Resultados}

A distribuição das condições socioeconômicas e demográficas dos familiares selecionados para o estudo está exposta na Tabela 1. Observa-se o predomínio de mulheres com baixa escolaridade (até o ensino fundamental incompleto) e idade entre 39 a 59 anos. $\mathrm{O}$ catolicismo foi à religião predominante, 57 (51,8\%), e 49 (44,5\%) dos sujeitos apresentaram uma renda familiar entre 1 a 2,5 salários mínimos ( $\mathrm{SM})$.

Em relação ao estigma, verifica-se, na Tabela 2 , que $66(60,0 \%)$ sujeitos revelaram preocupação em contrair a TB, no entanto, $100(90,9 \%)$ deles não sentiram vergonha do familiar doente, $84(76,4 \%)$ não esconderam da comunidade que 
Tabela 2. Distribuição dos escores de estigma associado à tuberculose na família, Ribeirão Preto (SP), 2011.

\begin{tabular}{|c|c|c|c|c|}
\hline \multirow[b]{2}{*}{ Questões de estigma } & \multicolumn{2}{|c|}{ Sim } & \multicolumn{2}{|c|}{ Não } \\
\hline & $\mathbf{n}$ & $\%$ & $\mathbf{n}$ & $\%$ \\
\hline Preocupação em contrair a TB & 66 & 60,0 & 44 & 40,0 \\
\hline O doente solicita segredo sobre seu status & 26 & 23,6 & 84 & 76,4 \\
\hline Vergonha do familiar por ele estar com TB & 10 & 9,1 & 100 & 90,9 \\
\hline Esconde das pessoas que ele(a) está doente & 26 & 23,6 & 84 & 76,4 \\
\hline Evita falar sobre TB na presença de outras pessoas & 44 & 40,0 & 66 & 60,0 \\
\hline Vergonha de sair com seu familiar doente & 4 & 3,6 & 106 & 96,4 \\
\hline Receio de ser visto no serviço de saúde onde ele(a) faz tratamento & 8 & 7,3 & 102 & 92,7 \\
\hline Utiliza expressões para substituir a palavra TB nas conversas com seu familiar doente & 15 & 13,7 & 95 & 86,3 \\
\hline Utiliza expressões para substituir a palavra TB nas conversas com amigos e vizinhos & 26 & 23,6 & 84 & 76,4 \\
\hline As pessoas da sua casa sabem que seu familiar está com TB & 103 & 93,6 & 7 & 6,4 \\
\hline
\end{tabular}

Fonte: Cálculo dos autores.

ele tinha TB e $66(60,0 \%)$ falam abertamente sobre a doença na presença de outras pessoas.

A análise de agrupamento permitiu a ordenação dos 110 sujeitos em quatro grupos (eixo das ordenadas), tendo-se considerado como ponto de corte a distância euclidiana de $20 \%$ (eixo das abscissas), denominados respectivamente de Grupo 1 (composto por 22 sujeitos, com índice de similaridade de 15\%), Grupo 2 (constituído por 39 sujeitos, com índice de similaridade de 10\%), Grupo 3 (formado por 23 sujeitos com índice de similaridade de $12 \%$ ) e Grupo 4 (constituído por 26 sujeitos, com índice de similaridade de $11 \%$ ) (Figura 1). O teste $\mathrm{T}^{2}$ de Hotelling mostrou diferença com significância estatística entre os grupos $(\mathrm{p}<0,001)$.

A Figura 2 mostra a representação gráfica das categorias das variáveis no mapa perceptual, com duas dimensões que representam $12,2 \%$ da variabilidade dos dados. A primeira que explica $6,4 \%$ dessa variabilidade e apresenta variáveis que correspondem às condições socioeconômicas e demográficas, acesso aos meios de informação e conhecimento sobre a doença. E a segunda dimensão, cuja contribuição foi menor (5,8\%), esteve relacionada às atitudes e comportamentos negativos dos familiares, exprimindo, portanto, $\mathrm{o}$ estigma social.

Com base no mapa, pode-se verificar que no lado esquerdo da dimensão 1 estão os grupos sem escolaridade ou com baixa escolaridade, sem acesso aos meios de informação e que pouco se informam sobre TB. Posicionados no lado direito dessa dimensão, estão as características opostas: níveis de escolaridade mais altos (ensino médio e superior concluídos), com acesso aos meios de informação e conhecimento sobre a doença.
A segunda dimensão divide os familiares segundo atitudes e comportamentos negativos associados ao estigma na TB. No lado negativo dessa dimensão, posicionam-se os sujeitos que sentiram vergonha do doente de TB, esconderam o status do seu familiar na comunidade e utilizaram expressões para substituir a palavra TB nas conversas com amigos e vizinhos. No lado oposto, as atitudes e comportamentos são vias positivas (Figura 2).

\section{Discussão}

Propôs-se investigar o estigma da TB nas famílias e identificar o perfil daquelas acometidas pelo evento em relação às condições socioeconômicas e demográficas. A partir das análises de agrupamento e correspondência múltipla foi possível verificar diferentes grupos e assim reconhecer a complexidade do objeto em estudo. Tais apontamentos refletem conclusões distintas e relevantes para a população analisada.

A vergonha do familiar doente, o encobrimento da doença na comunidade e o uso de expressões para substituírem a palavra TB nas conversas com o familiar, amigos e vizinhos foram comportamentos e atitudes identificados nos Grupos 1 e 2, estando em correspondência com a baixa escolaridade, o acesso incipiente aos meios de informação e pouca mobilização face às razões e compreensões sobre a doença.

No que refere ao nível educacional, estudos foram concordantes. Apesar de guardar diferenças metodológicas, estes apontaram a baixa escolaridade como condição social dos doentes acometidos pelo estigma na $\mathrm{TB}^{4,27}$. Outras pesquisas 


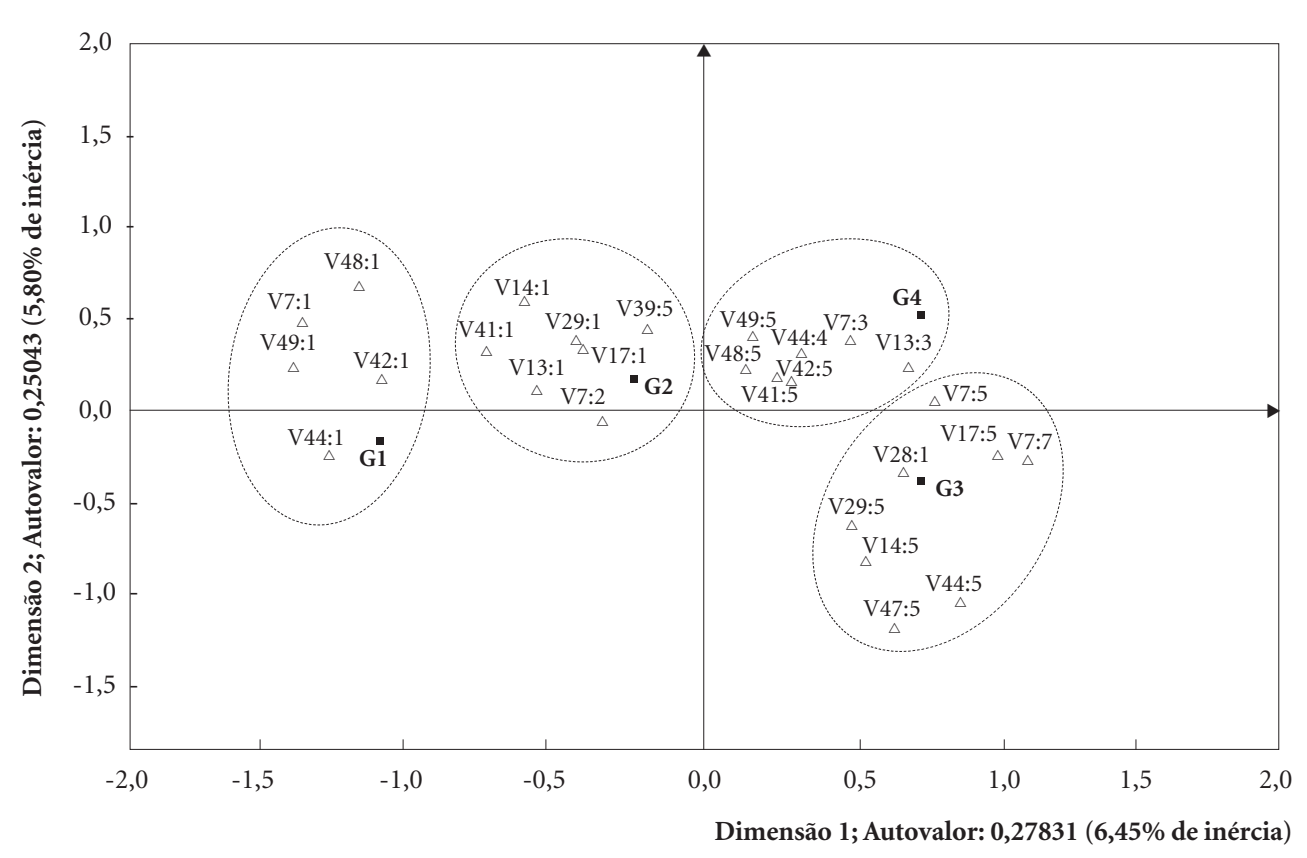

Legenda: G1 - Grupo 1:V7:1 - Sem escolaridade; V42:1 - Esconde das pessoas que o familiar tem TB; V44:1 - Evita falar sobre TB na presença de outras pessoas; V48:1 - Utiliza expressões para substituir a palavra TB nas conversas com seu familiar doente; V49:1 - Utiliza expressões para substituir a palavra TB nas conversas com amigos e vizinhos; G2 - Grupo 2: V7:2 - Ensino Fundamental Incompleto; V13:1 - Não tem o hábito de ler jornal; V14:1 - Não tem o hábito de ler revista; V17:1 - Não tem o hábito de acessar a internet; V29:1 - Procura saber pouco sobre TB; V39:5 - Não tem preocupação em contrair a TB; V41:1 - Tem vergonha do familiar por ele estar com TB; G3 - Grupo 3: V7:5 - Ensino Médio completo; V7:7 - Ensino Superior Completo; V14:5 - Tem o hábito de ler revista; V17:5 - Tem o hábito de acessar a internet; V28:1 - Tem conhecimento sobre TB; V29:5 - Procura saber muito sobre TB; V44:5 - Fala abertamente sobre TB na presença de outras pessoas; V47:5 - Não tem receio de ser visto no serviço de saúde onde seu familiar faz tratamento; G4 - Grupo 4: V7:3 - Ensino Fundamental completo; V13:3 - Às vezes lê jornal; V41:5 - Não tem vergonha do familiar por ele estar com TB; V42:5 - Não esconde das pessoas que o familiar tem TB; V44:4 - Fala sobre TB na presença de outras pessoas; V48:1 - Não utiliza expressões para substituir a palavra TB nas conversas com seu familiar doente; V49:1 - Não utiliza expressões para substituir a palavra TB nas conversas com amigos e vizinhos.

Figura 2. Categorias dos grupos de estigma social e das características socioeconômicas, demográficas e de conhecimento sobre a doença, Ribeirão Preto (SP), 2011.

também corroboram esses resultados, uma vez que identificaram a percepção do contágio da TB como uma das principais causa da estigmatização e ressaltam que o desconhecimento a respeito da doença pode intensificar as atitudes discriminatórias na família e na comunidade ${ }^{8-10,28,29}$.

Segundo autores ${ }^{30}$ o estigma possui um substrato sociocultural cujos sujeitos, que não têm conhecimento sobre a TB, estão expostos a comportamentos negativos e a atitudes discriminatórias. Observa-se que doente e família convivem com o medo da exclusão social e, dessa forma, evitam revelar seu status e falar sobre a doença no trabalho e na comunidade ${ }^{3,4,8,10}$. A substituição da palavra "tuberculose" por outros termos e expressões pode ser um meio de suavizar a sua condição e não revelar o diagnóstico para as pessoas do seu convívio.
Apesar desse comportamento, a preocupação das famílias em relação à discriminação que afeta seu familiar são relatadas nesses mesmos estudos, portanto, falar abertamente sobre a TB na comunidade e nos serviços de saúde em que o doente realiza o tratamento torna-se uma questão complexa e desafiadora, pois pode impedi-lo de ter apoio e acolhimento em suas relações sociais. Esta situação é vista por autores ${ }^{7,31}$ como uma dialética entre o desejo da família em ajudar o doente e a manutenção/permanência do estigma, que por vezes não é intencional, mas está associada às crenças e valores instituídos.

Nesse contexto, verifica-se a tensão existente entre a ênfase individual e a social, como algo que se constrói e reconstrói não só através do tempo, mas também do espaço, ou seja, das diferenças entre grupos sociais com suas representações e da 
heterogeneidade das perspectivas e práticas que se encontram dentro de um grupo aparentemente homogêneo (pessoas acometidas pela TB) ${ }^{32}$.

Tal heterogeneidade de perspectivas e representações em relação à TB se mostrou evidenciada nos Grupos 3 e 4, com perfis bem diferenciados em relação aos Grupos 1 e 2. Nestes grupos, comportamentos e atitudes positivas foram observadas no convívio com o doente e a comunidade. Concomitantemente, apresentaram níveis de escolaridade mais elevados, informaram acessar continuamente a internet, leem revistas, buscam outras fontes de informação, como rádio e televisão, e se dizem conhecedores da TB. Observa-se que o acesso à informação e a alta escolaridade desses grupos superou os mitos e as crenças ressonantes na sociedade, e por isso falam abertamente sobre a doença e reconhecem a possibilidade de cura.

Certamente, quando o estigma da TB passar a ser reconhecido e compreendido como um problema de saúde pública pelas equipes, autoridades e sociedade ter-se-ão medidas mais efetivas para seu enfrentamento ${ }^{33}$. Nesta perspectiva, os resultados desta pesquisa apontam dois grupos marcados pelo estigma e pela desigualdade social e que, portanto, demandam ações prioritárias em saúde.

Ressalta-se que tais ações devem ser direcionadas à conjuntura social e à realidade local das famílias, no sentido de compartilhar conhecimentos e práticas relacionadas ao cuidado do doente de $\mathrm{TB}$, sendo relevante o trabalho de uma equipe multiprofissional que esteja sensibilizada com a problemática em questão e que discuta intervenções desde o aspecto biológico ao social da doença.

Dentre outras medidas necessárias, destacase o reforço à intersetorialidade entre a saúde e a educação, com finalidade de ressocialização, de escolarização e de novas oportunidades de geração de renda e de trabalho. Ademais, ressalta-se a mobilização social, através do preparo e do engajamento da sociedade para a reivindicação de soluções, próximas a sua realidade, e desenvolvimento de ações coletivas que estejam empoderadas e sensíveis ao problema da $\mathrm{TB}^{34,35}$.

No Brasil, este envolvimento comunitário nas ações de controle da doença é bastante recente.
A formulação de diretrizes de fortalecimento da participação comunitária nos âmbitos municipal e estadual tem sido subsidiada pela estratégia denominada Comunicação, Advocacia e Mobilização Social (CAMS), que consiste no acesso à informação de forma compartilhada, baseandose na transparência e no respeito aos aspectos culturais, assim como nas diferenças entre as populações, com a finalidade de realização de ações individuais e de grupos organizados que devem se mobilizar e reivindicar seus direitos, diante de suas necessidades de saúde ${ }^{36}$.

Nesta perspectiva, segundo o Programa Nacional de Controle da Tuberculose (PNCT), o desenvolvimento de ações baseadas na estratégia CAMS deve estar presente nas propostas dos programas municipais e estaduais de controle da TB, contribuindo para a realização do diagnóstico precoce, a adesão ao tratamento, o combate ao estigma e à discriminação e o fortalecimento da participação comunitária nos Conselhos de Saúde e em suas respectivas conferências ${ }^{34}$.

As limitações encontradas no estudo se referem à utilização do instrumento submetido apenas à validação semântica e de conteúdo, o que pode ter gerado um viés de aferição na obtenção dos dados. Além disso, a relação entre a representação histórico-social da doença e o estigma não foi possível de ser mensurada, contudo, outras investigações de cunho qualitativo poderão contribuir para a compreensão da ocorrência do estigma na família.

Por se tratar de técnicas estatísticas de caráter exploratório, a relação de causalidade a partir da correspondência entre as observações, não pôde ser estabelecida. Entretanto, foi possível identificar inter-relações entre as características socioeconômicas, demográficas e de conhecimento sobre a doença e o estigma social, definindo os perfis de grupos de familiares.

Diante da relevância de se investigar o perfil das famílias que são acometidas pelo estigma da TB e das evidências quanto à ocorrência deste fenômeno na dinâmica familiar, este estudo traz uma contribuição considerável para a saúde coletiva e aponta para a necessidade de se alçar novos patamares no que se refere à eliminação de doenças infecciosas e emblemáticas da pobreza, como a TB. 


\section{Colaboradores}

MM Touso participou da concepção do projeto, análise e interpretação dos dados e redação do artigo. MP Popolin, JA Crispim, IM Moreira e AS Ferraudo participaram da análise e interpretação dos dados e da redação do artigo. LBB Rodrigues, M Yamamura, IC Pinto, AA Monroe, PF Palha e TCS Villa participaram da redação do artigo e revisão crítica relevante do conteúdo intelectual. RA Arcêncio participou da concepção do projeto, análise e interpretação dos dados, redação do artigo, revisão crítica relevante do conteúdo intelectual e aprovação da versão.

\section{Agradecimentos}

Ao CNPq pelo financiamento. A FAPESP pelas bolsas de Mestrado e Iniciação Científica.

\section{Referências}

1. World Health Organization (WHO). Global tuberculosis report 2012. Geneva: WHO; 2012.

2. Brasil. Ministério da Saúde (MS). Secretaria de Vigilância em Saúde. Boletim Epidemiológico. Brasília: MS; 2013.

3. Coreil J, Mayard G, Simpson KM, Lauzardo M, Zhu Y, Weiss MG. Structural forces and the production of TBrelated Stigma among Haitians in two contexts. Soc Sci Med 2010; 71(8):1409-1417.

4. Kipp AM, Pungrassami P, Nilmanat K, Sengupta S, Poole C, Strauss RS, Chongsuvivatwong V, Van Rie A. Socio-demographic and AIDS-related factors associated with tuberculosis stigma in southern Thailand: a quantitative, cross-sectional study of stigma among patients with TB and healthy community members. BMC Public Health 2011; 11:675.

5. Goffman E. Estigma: notas sobre a manipulação da identidade deteriorada. $4^{\mathrm{a}}$ ed. Rio de Janeiro: Guanabara Koogan; 1988.

6. Ronzani TM, Furtado EF. Estigma social sobre o uso de álcool. J Bras Psiquiatr 2010; 59(4):326-332.

7. Macq J, Solis A, Martinez G, Martiny P, Dujardin B. An exploration of the social stigma of tuberculosis in five "municipios" of Nicaragua to reflect on local interventions. Health Policy 2005; 74(2):205-217.

8. Baral SC, Deepak KK, Newell JN. Causes of stigma and discrimination associated with tuberculosis in Nepal: a qualitative study. BMC Public Health 2007; 7:211.

9. Somma D, Thomas BE, Karim F, Kemp J, Arias N, Gosoniu GD, Abouihia A, Weiss MG. Gender and sociocultural determinants of TB-related stigma in Bangladesh, India, Malawi and Colombia. Int J Tuberc Lung Dis 2008; 12(7):856-866.

10. Dodor EA, Kelly S. We are afraid of them': Attitudes and behaviours of community members towards tuberculosis in Ghana and implications for TB control efforts. Psychol Health Med 2009; 14(2):170-179.

11. Long NH, Johansson E, Diwan VK, Winkvist A. Fear and social isolation as consequences of tuberculosis in Vietnam: a gender analysis. Health Policy 2001; 58(1):69-81.

12. Eastwood SV, Hill PC. A gender-focused qualitative study of barriers to accessing tuberculosis treatment in the Gambia, West Africa. Int J Tuberc Lung Dis 2004; 8(1):70-75.

13. Pôrto A. Representações sociais da tuberculose: estigma e preconceito. Rev Saude Publica 2007; 41(Supl. 1):43-49.

14. Sousa AS, Kantorski LP, Bielemann VLM. A AIDS no interior da família: percepção, silêncio e segredo na convivência social. Acta sci Health sci 2004; 26(1):1-9. 
15. Weiss MG, Ramakrishna J. Stigma interventions and research for international health. Lancet 2006; 367:536538.

16. Brasil. Ministério da Saúde (MS). Secretaria de Vigilância em Saúde. Programa Nacional de Controle da Tuberculose. Brasília: MS; 2004

17. Fundação Sistema Estadual de Análise de Dados (Seade). Índice Paulista de Responsabilidade Social. São Paulo: Seade; 2008.

18. Instituto Brasileiro de Geografia e Estatística (IBGE). Estimativa da população 2011. [acessado 2012 abr 27] Disponível em: http://www.ibge.gov.br/home/estatística/população/estimativa2011/POP2011_DOU.pdf

19. São Paulo. Secretaria Estadual de Saúde. Sistema Estadual de Notificação da Tuberculose (TBWEB). [acessado 2011 jul 02]. Disponível em: http://www.cvetb.saude. sp.gov.br

20. Macq J, Solis A, Martinez G. Assessing the stigma of tuberculosis. Psychol Health Med 2006; 11(3):346-352.

21. Van Rie A, Sengupta S, Pungrassami P, Balthip Q, Choonuan S, Kasetjaroen Y, Strauss RP, Chongsuvivatwong V. Measuring stigma associated with tuberculosis and HIV/AIDS in southern Thailand: exploratory and confirmatory factor analyses of two new scales. Trop Med Int Health 2008; 13(1):21-30.

22. Weiss MG, Somma D, Karim F, Abouihia A, Auer C, Kemp J. Cultural epidemiology of TB with reference to gender in Bangladesh, India and Malawi. Int J Tuberc Lung Dis 2008; 12(7):837-847.

23. Mingoti SA. Análise de dados através de métodos de estatística multivariada: uma abordagem aplicada. Belo Horizonte: Editora UFMG; 2005.

24. Morrison DF. Multivariate Statistical Methods. New York: Mc-Graw-Hill; 1990. [acessado 2014 set 9]. Disponível em: https://stat.duke.edu/courses/Spring10/ sta345/morrison/Mori1990a.pdf

25. Alves SMF, Fernandes PM, Reis EF. Análise de correspondência como instrumento para descrição do perfil de trabalhadores da cultura de tomate de mesa em Goiás. Ciência Rural 2009; 39(7):2042-2049.

26. Hair Junior JF, Anderson RE, Tatham RL, Black WC. Análise multivariada de dados. $5^{\mathrm{a}}$ ed. Porto Alegre: Editora Bookman; 2005.

27. Abioye IA, Omotayo MO, Alakija W. Socio-demographic determinants of stigma among patients with pulmonary tuberculosis in Lagos, Nigeria. Afr Health Sci 2011; 11(Supl. 1):100-104.
28. Sengupta S, Pungrassami P, Balthip Q, Strauss R, Kasetjaroen Y, Chongsuvivatwong V, Van Rie A. Social impact of tuberculosis in southern Thailand: views from patients, care providers and the community. Int $\mathrm{J} \mathrm{Tu}$ berc Lung Dis 2006; 10(9):1008-1012.

29. Atre S, Kudale A, Morankar S, Gosoniu D, Weiss MG Gender and community views of stigma and tuberculosis in rural Maharashtra, India. Glob Public Health 2011; 6(1):56-71.

30. Abebe G, Deribew A, Apers L, Woldemichael K, Shiffa J, Tesfaye M, Abdissa A, Deribie F, Jira C, Bezabih M, Aseffa A, Duchateau L, Colebunders R. Knowledge, Health Seeking Behavior and Perceived Stigma towards Tuberculosis among Tuberculosis Suspects in a Rural Community in Southwest Ethiopia. PloS One 2010; 5(10):e13339.

31. Freitas IM, Crispim JA, Pinto IC, Villa TCS, Brunello MEF, Pinto PFPS, Arcêncio RA. Conhecimento e percepção sobre tuberculose das famílias de pacientes em tratamento diretamente observado em um serviço de saúde de Ribeirão Preto-SP, Brasil. Texto Contexto Enferm 2012; 21(3):642-649.

32. Gonçalves H. A tuberculose ao longo dos tempos. Hist. Cienc. Saúde-Manguinhos 2000; 7(2):303-325.

33. Marquieviz J, Alves IS, Neves EB, Ulbricht L. A Estratégia de Saúde da Família no Controle da tuberculose em Curitiba (PR). Cien Saude Colet 2013; 18(1):265-271.

34. Santos Filho ET, Santos ZMG. Estratégias de controle da tuberculose no Brasil: articulação e participação da sociedade civil. Rev Saude Publica 2007; 41(Supl. 1):111-116

35. Barros MBA, Francisco PMSB, Zanchetta LM, Cesar CLG. Tendências das desigualdades sociais e demográficas na prevalência de doenças crônicas no Brasil, PNAD: 2003- 2008. Cien Saude Colet 2011; 16(9):37553768.

36. Brasil. Ministério da Saúde (MS). Programa Nacional de Controle da Tuberculose. Manual de Recomendações para o Controle da Tuberculose no Brasil. Brasília: MS; 2011.

Artigo apresentado em 04/09/2013

Aprovado em 20/10/2013

Versão final apresentada em 23/10/2013 
